\title{
Hodgkin's disease presenting as liver abscesses
}

\author{
A Zaman, P N Bramley, J Wyatt, A Simmons, M S Losowsky, R Bolton
}

\begin{abstract}
A patient is reported who presented with sterile hepatic abscesses which proved to be the first manifestation of Hodgkin's disease. He failed to improve with antibiotic treatment but responded to combined chemotherapy. Liver involvement as the first sign of Hodgkin's disease is rare. Liver abscesses due to Hodgkin's disease seem to be previously unreported.
\end{abstract}

\section{Case report}

A 29 year old man began to feel unwell and tired in September 1987. Initial investigations in December showed a neutrophil leucocytosis: white cell count $24.5 \times 10^{9} / 1$ with $87 \%$ neutrophils, raised erythrocyte sedimentation rate 57 $\mathrm{mm}$ in the first hour (normal $<20$ ), and abnormal liver function. Alkaline phosphatase was raised at 6.1 KAU/l (normal 1.3-3.6) and $\gamma$ glutamyltransferase at $1.5 \mathrm{KAU} / 1$ (normal $0 \cdot 1$ $0 \cdot 7)$.

He was referred to hospital. His symptoms were unchanged and clinical examination was negative. Abdominal ultrasound showed hepatomegaly with fluid filled lesions and compression of a patent portal vein. Diagnoses considered were intra-abdominal malignancy, hepatic abscess, and angiosarcoma. The patient had not received antibiotic drugs at this point.

Guided liver biopsy produced necrotic material; Gram stain examination was negative. The material was found to be sterile after aerobic, anaerobic, and fungi cultures using standard methods. Histology showed purulent debris, with dense fibrosis and an occluded portal vein radicle in the adjacent liver lobule (Fig 1). After the biopsy he was pyrexial for 24 hours and was treated with intravenous tobramycin and cefotaxime for 14 days. A repeat ultrasound scan showed no change.

He was referred to St James's University Hospital in March 1988. On admission he was unwell, had lost weight, and was having sweats and rigors. He was pyrexial and jaundiced with an enlarged tender liver. Ultrasound showed low echogenic areas containing fluid and gas consistent with liver abscesses. Aspiration was repeated, and once again Gram stain, aerobic, anaerobic, and fungal cultures were negative. His white cell count remained high at $33 \times 10^{9} / 1$; blood cultures were negative.

$\mathrm{He}$ was treated with penicillin and metronidazole for 26 days. There was no improvement in his condition and he remained pyrexial. In the next six weeks his liver lesions were drained surgically on two occasions. Small quantities of sterile pus and further liver biopsy samples were obtained each time. Repeat ultrasound suggested resolving abscesses. There was some improvement in his overall state and so he was discharged from hospital.

At outpatient review it was clear that he had deteriorated as he complained of generalised itching and sweating. Examination now showed right axillary lymphadenopathy, skin excoriations, and a temperature of $37.5^{\circ} \mathrm{C}$. He had become anaemic, haemoglobin $9.7 \mathrm{~g} / 1$; white cell count $27.4 \times 10^{9} / 1$; plasma viscosity $1.94 \mathrm{cP}$
Figure 1: Liver biopsy showing fibrosis engulfing foci of residual hepatocytes on the left; on the right there is a dense mixed

inflammatory cell infiltrate, which includes many eosinophils, and which has destroyed the liver architecture. (Haematoxylin and eosin; original magnification $\times 32$.)

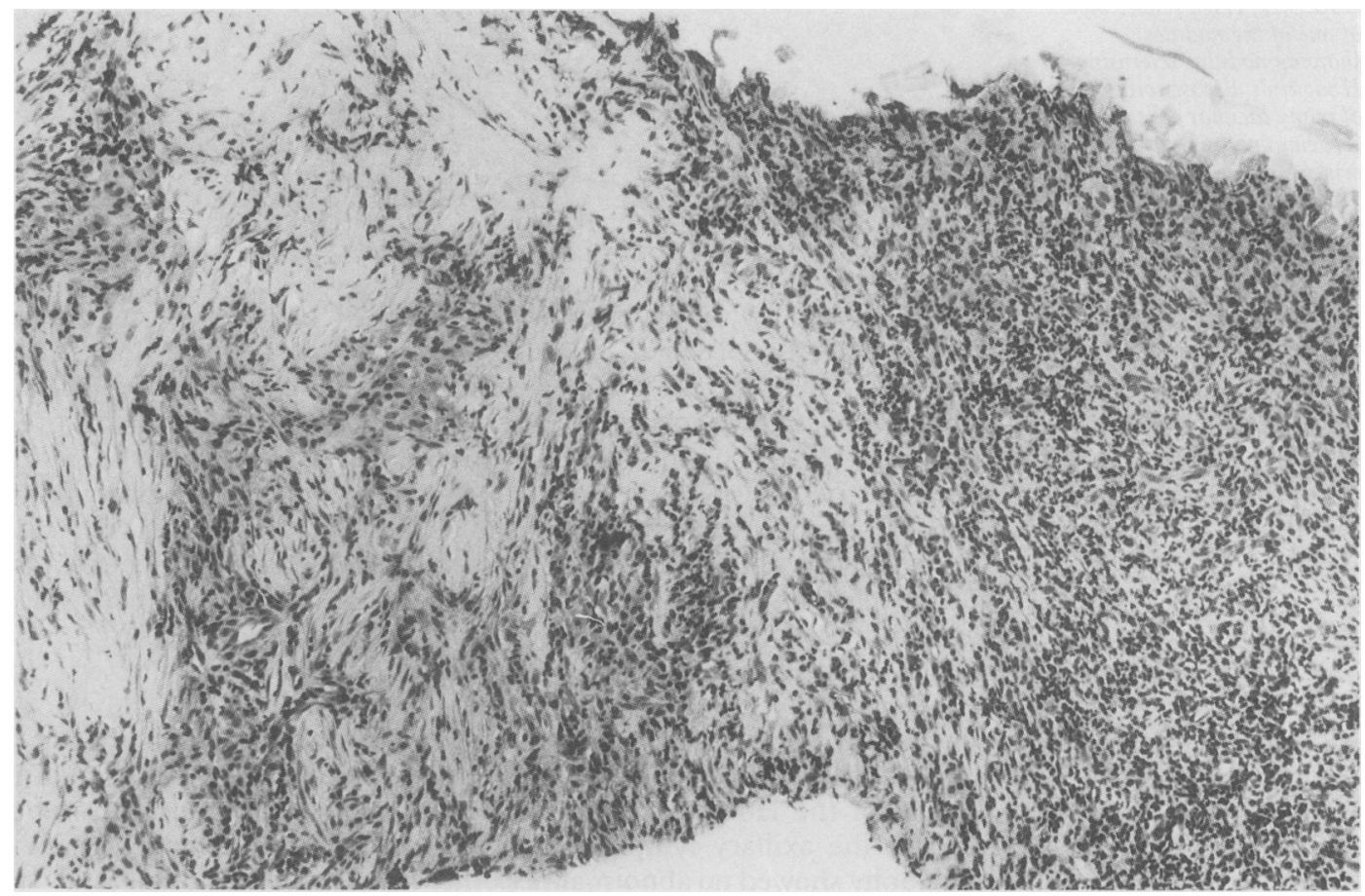


Figure 2: (A) Axillary lymph node: bands of fibrosis divide the tissue into distinct nodules. (Reticulin stain; original magnification $\times 13$.) $(B)$ High power view of one of the nodules showing nodular sclerosing Hodgkin's disease with one of many lacunar cells. (Haematoxylin and eosin; original magnification $\times 50$.)
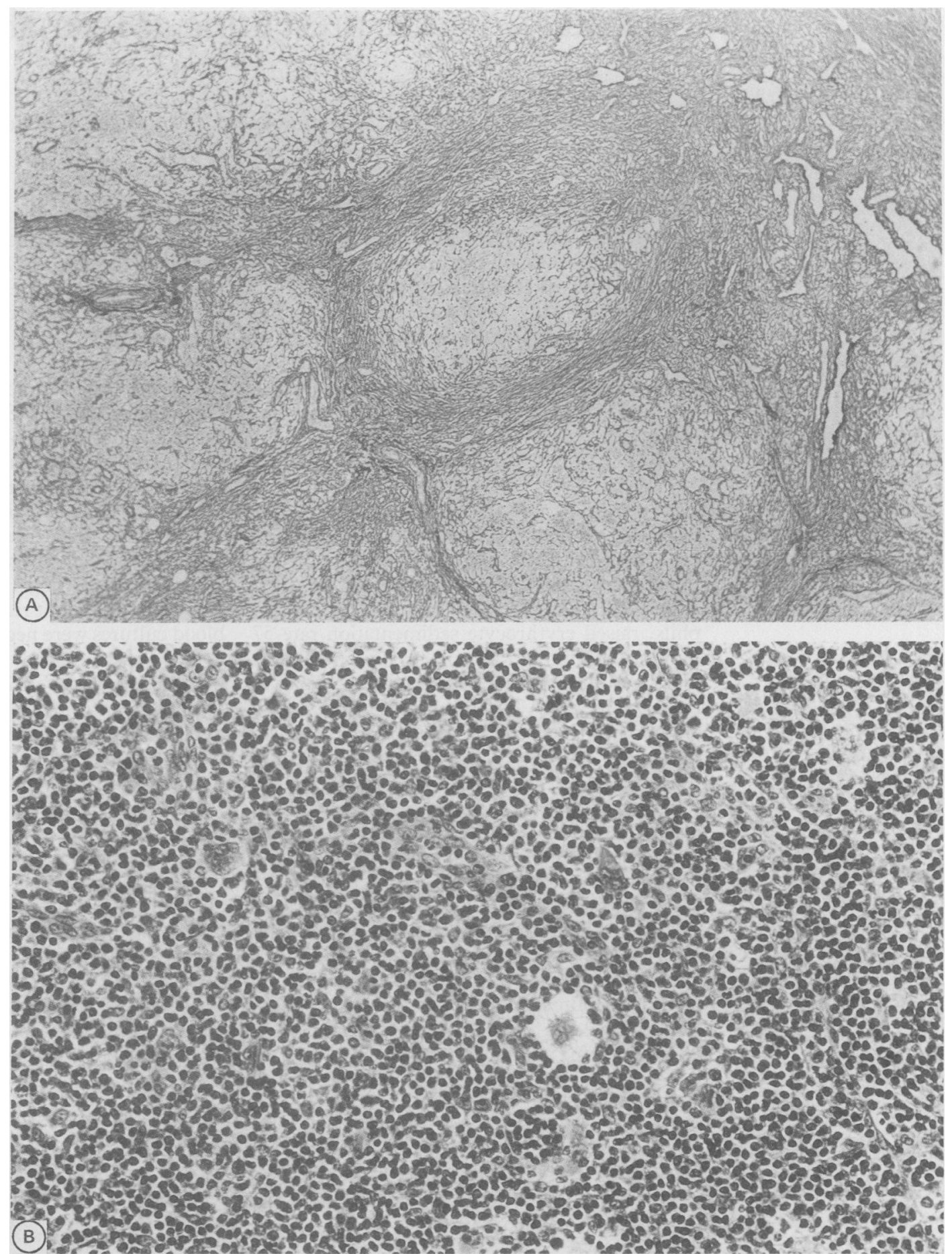

(normal 1.56-1.73); liver function tests: bilirubin $30 \mu \mathrm{mol} / 1$ (normal 3-15), alanine transaminase $12 \mathrm{IU} / 1$ (normal 0-35), alkaline phosphatase 46.4 KAU/dl (normal 3-13); albumin 33 g/l (normal 37-49), and total protein $67 \mathrm{~g} / 1$ (normal 61-77).

He was readmitted to hospital, and a right axillary lymph node biopsy showed classical nodular sclerosing Hodgkin's disease (Fig 2). Further sections were examined from the previous liver biopsy and these showed occasional Reed-Sternberg cells (Fig 3), confirming liver involvement by Hodgkin's disease. Areas of necrosis with heavy polymorph infiltration were a feature of the Hodgkin's disease in both the liver and the axillary lymph node. Computed tomography showed no abnormalities other than the liver lesions and the right axillary lymphadenopathy. Bone scan was normal, and marrow trephine showed reactive changes only.

At the end of November 1988 treatment was started with the combination of chlorambucil, vincristine, procarbazine, and prednisolone. $\mathrm{He}$ rapidly improved, and his alkaline phosphatase, white cell count, and platelets rapidly fell towards normal, while his albumin returned to normal (Fig 4). varies from necrosis of individual Reed-Sternberg cells to massive necrosis of entire lymph nodes.' Stansfield states: 'Solid clusters of

\section{Discussion}

Necrosis is common in Hodgkin's disease and 
Figure 3: Liver biopsy shown in Figure 1. When multiple levels were examined occasional ReedStermberg cells were apparent in the inflammatory cell areas. (Haematoxylin and eosin; original magnification $\times 65$.

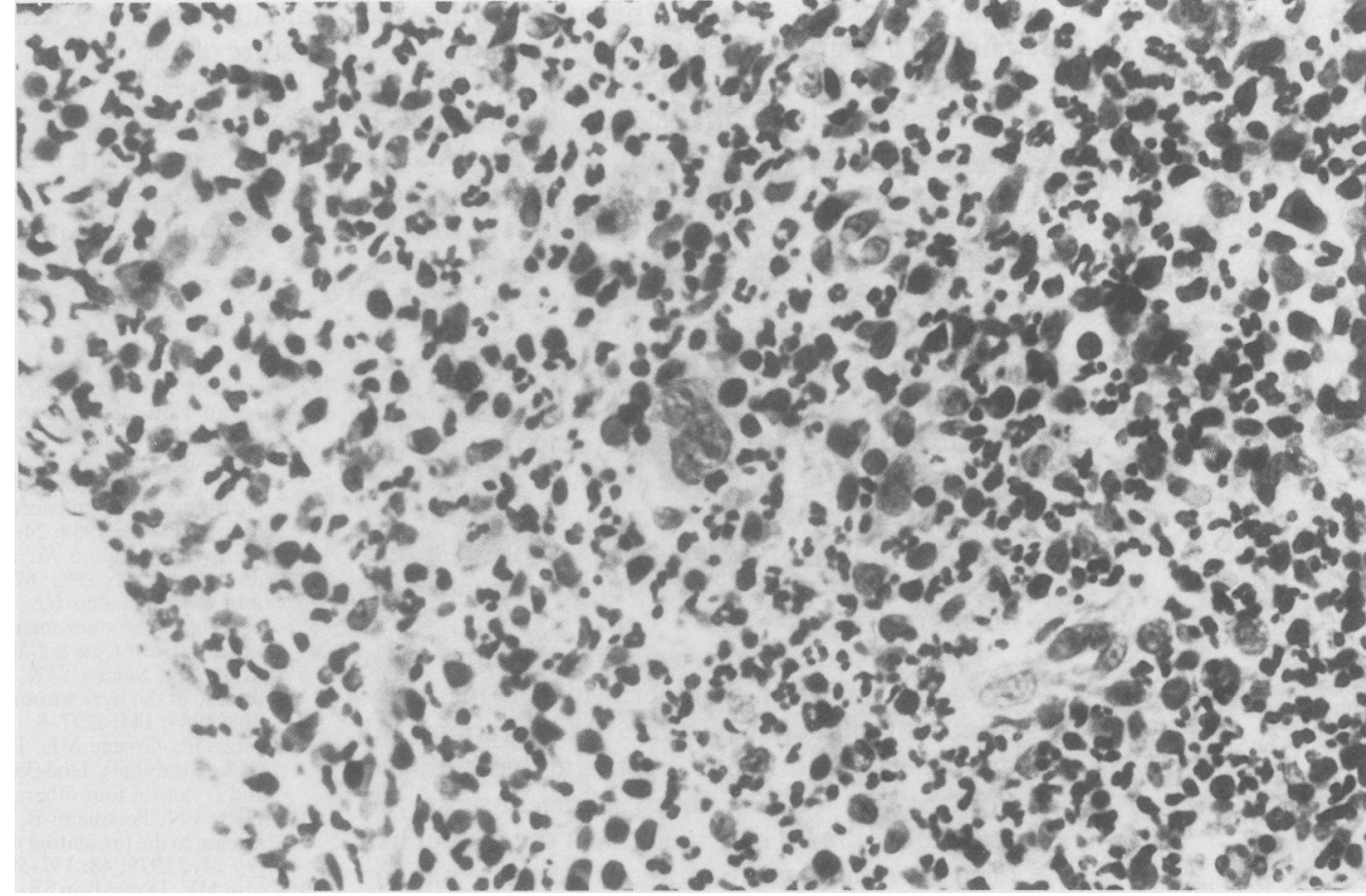

lacunar cells are particularly liable to undergo necrosis and when this is accompanied, as it often is, by heavy polymorph infiltration, the lesions may be mistaken for abscesses and the true nature of the process may be overlooked." Nodular sclerosing Hodgkin's disease may provoke an inflammatory response in the affected tissues which is independent of the extent or even the presence of necrosis. Whereas Hodgkin's disease is not in itself a suppurative condition, we are persuaded that abscesses may occur without the presence of superimposed infection.

The negative cultures before antibiotic treatment and the similar pattern of the characteristic histological process occurring in the liver and the second lymph node biopsy (of a subsequent right inguinal swelling) suggest that the liver abscesses

$\rightarrow-$ White cell count $\quad-+-$ Alkaline phosphatase

*. Albumin

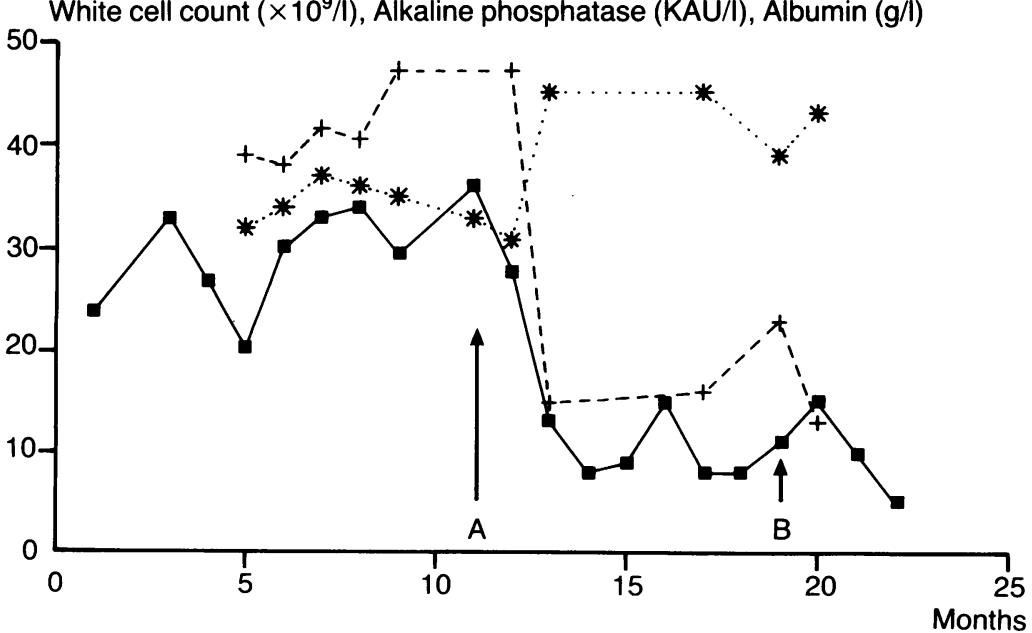

Figure 4: Sequential changes in blood tests since presentation, showing initiation of chemotherapy and response to treatment. were part of Hodgkin's disease and not primarily or secondarily infective. The continued failure to show any organism, the failure of prolonged antibiotic treatment, and the dramatic rapid clinical and subsequent radiological improvement after combined chemotherapy further supports this conclusion.

Primary presentation of Hodgkin's disease in extranodal sites is unusual, occurring in less than $5 \%$ of cases. Selby and McElwain ${ }^{2}$ state that 'it should be emphasized that when the diagnosis of $\mathrm{HD}$ is made on the basis of a biopsy taken from a site other than lymph node and there is no lymphadenopathy, the first response should be to review that diagnosis critically and cautiously - it may well not be HD.'

On the other hand, eventual involvement of the liver is not uncommon. Usually there is generalised infiltration of the liver. The common route of progression is from lymph nodes to the spleen and then to the liver. Glatstein $e t a l^{3}$ concluded that liver involvement does not occur without concomitant splenic involvement. This view is echoed by Appelqvist and $\mathrm{Salmo}^{4}$ and Gobbi et al. ${ }^{5} \mathrm{~A}$ patient with liver but not splenic involvement was reported by Gordon et $a l^{6}$ in 1984. A 67 year old woman with stage IIIA nodular sclerosing Hodgkin's disease had two foci of Hodgkin's disease present in a wedge biopsy specimen of liver. After splenectomy serial sectioning of the spleen failed to show macroscopic or microscopic evidence of Hodgkin's disease.

The patient reported here did not have clinical splenomegaly, and the spleen was not enlarged on ultrasound or computed tomographic examination. Histology of the spleen is not available. We cannot be sure that the spleen was free from disease as a normal size spleen does not preclude involvement.

Hodgkin's disease can be associated with liver problems in other ways. These include cholestatic jaundice (occurring in the absence of detect- 
able Hodgkin's disease, ${ }^{7}$ hepatitis or hepatic failure ${ }^{8}$ and granulomatous hepatitis. ${ }^{9}$ In a study of 185 patients with Hodgkin's disease, Kadin et al found isolated tubercle like granulomata in otherwise uninvolved organs in 23 patients. In three patients with granulomata in the liver there was no splenic involvement. Kadin et al did not consider that the presence of granulomata indicated involvement of the liver with Hodgkin's disease.

A search of published reports showed no case of Hodgkin's disease presenting as liver abscesses. Trewby et $a l^{8}$ reported a patient referred with a diagnosis of liver abscess but subsequent isotope scan and computed tomography did not show an abscess cavity or filling defect in the liver. The patient responded to chemotherapy.

Liver abscesses are usually associated with pyogenic infection. Modern management by means of percutaneous needle aspiration and drainage and antibiotic treatment has reduced mortality to $1 \cdot 5 \% .^{10}$ In pyogenic abscess the material aspirated may be sterile if the patient has been pretreated with antibiotics. In our patient diagnostic features of Hodgkin's disease were present on review of step sections of the liver biopsy specimen. Multiple levels were not initially examined because of the clear clinical picture of abscesses.

The present case suggests that Hodgkin's disease should be considered as a possible cause in any patient with a hepatic abscess from which sterile material has been obtained and who does not respond to adequate antibiotic treatment.

1 Stansfield AG. In: Lymph node biopsy interpretation. Edinburgh: Churchill Livingstone, 1985: 211.

2 Selby P, McElwain TJ. Hodgkin's disease. Oxford: Blackwell Scientific, 1987.

3 Glatstein E, Gwensey JM, Rosenberg SA. The value of laparotomy and splenectomy in the staging of Hodgkin's disease. Cancer 1969; 24: 709-18

4 Appelqvist P, Salmo M. Staging laparotomy in Hodgkin's disease. Int Surg 1982; 67: 251-5.

5 Gobbi PG, Parinello GA, Di Pruslo U, et al. New clinical criteria for the assessment of liver involvement in Hodgkin's disease. Eur F Cancer Clin Oncol 1982; 18: 1243-9.

6 Gordon CD, Sidawy MK, Talarico L, Kondi E. Hodgkin's disease in the liver without splenic involvement. Arch Intern disease in the liver with

7 Perera DR, Greene ML, Fenster LF. Cholestasis associated with extrabiliary Hodgkin's disease. Report of three cases with extrabiliary Hodgkin's disease. Report of three cases
and review of four others. Gastroenterology $1974 ; 67: 680-5$.

8 Trewby PN, Portmann B, Brinkley DM, Williams R. Liver disease as the presenting manifestation of Hodgkin's disease. Q F Med 1979; 48: 137-50.

9 Kadin ME, Donaldson SS, Dorfman RF. Isolated granuloma in Hodgkin's disease. $N$ Engl F Med 1970; 283: 859-61

10 Sheinfield AM, Steiner AE, Rivkin LB, et al. Transcutaneous drainage of abscesses of the liver guided by computed tomography. Surg Gynecol Obstet 1982;155: 662-6. 